\title{
Answer to the rhythm puzzle
}

\author{
Z. Li $\cdot$ F. Shang $\cdot$ W. Zhang $\cdot$ Y. Wang
}

Published online: 12 February 2011

(C) Springer Media/Bohn Stafleu van Loghum 2011

The 12-lead ECG shows the Wolff-Parkinson-White (WPW) syndrome (Fig. 1, colour panel, black arrow: delta wave) and premature ventricular contractions (PVCs). The PVCs come from the right ventricular outflow according to the electrocardial vector (Fig. 1, colour panel, red arrow).

There were two different types of onset ECG with fast and regular rhythm: one with normal QRS complexes was an orthodromic supraventricular tachycardia (SVT) with a cycle length of $380 \mathrm{~ms}$, which the impulse conducted retrogradely through the accessory pathway (Fig. 1, black panel, thick red arrow), and the other with wide QRS complexes was ventricular tachycardia (VT, Fig. 1, black panel, thin red arrow). The SVT was evoked easily by PVCs or paroxysmal VT, and it was confirmed by the
Holter monitoring (Fig. 1, black panel). So, the diagnosis is WPW syndrome and SVT triggered by paroxysmal VT.

The accessory pathway located at 3 o'clock of the mitral valve annulus was successfully ablated with radiofrequency (Fig. 2, left panel, black arrow: delta wave, red arrow: normal). The target map showed that the $\mathrm{PVC}$ at $8 \mathrm{~mm}$ of the big tip catheter was $43 \mathrm{~ms}$ earlier than any other leads (Fig. 2, right panel, red arrow), and the original site of the PVCs at the right ventricular outflow was successfully ablated with radiofrequency. The drawing shows the ablated site of VT (Fig. 3, right panel, asterisk: ablated site) and the orthodromic circus movement tachycardia (left panel). She is asymptomatic after 3 months of follow-up.
Z. Li $(\bowtie) \cdot$ F. Shang $\cdot$ W. Zhang $\cdot$ Y. Wang

Department of Cardiology, Tangdu Hospital, Fourth Military

Medical University,

Shaanxi 710038, China

e-mail: lizhili@fmmu.edu.cn 
Fig. 1 Colour panel the usual 12-lead surface ECG. Paper speed is as usual $(25 \mathrm{~mm} / \mathrm{s})$, and voltage is half the usual $(5 \mathrm{mv} / \mathrm{mm})$. Black arrow $=$ delta wave, red arrow $=$ premature ventricular contraction. Black panel the significant episode documented by Holter monitoring. Thin black arrow $=$ delta wave, thin red arrow $=\mathrm{VT}$, thick red arrow $=$ SVT. Paper speed is as usual $(25 \mathrm{~mm} / \mathrm{s})$, and voltage is as usual $(10 \mathrm{mv} / \mathrm{mm})$

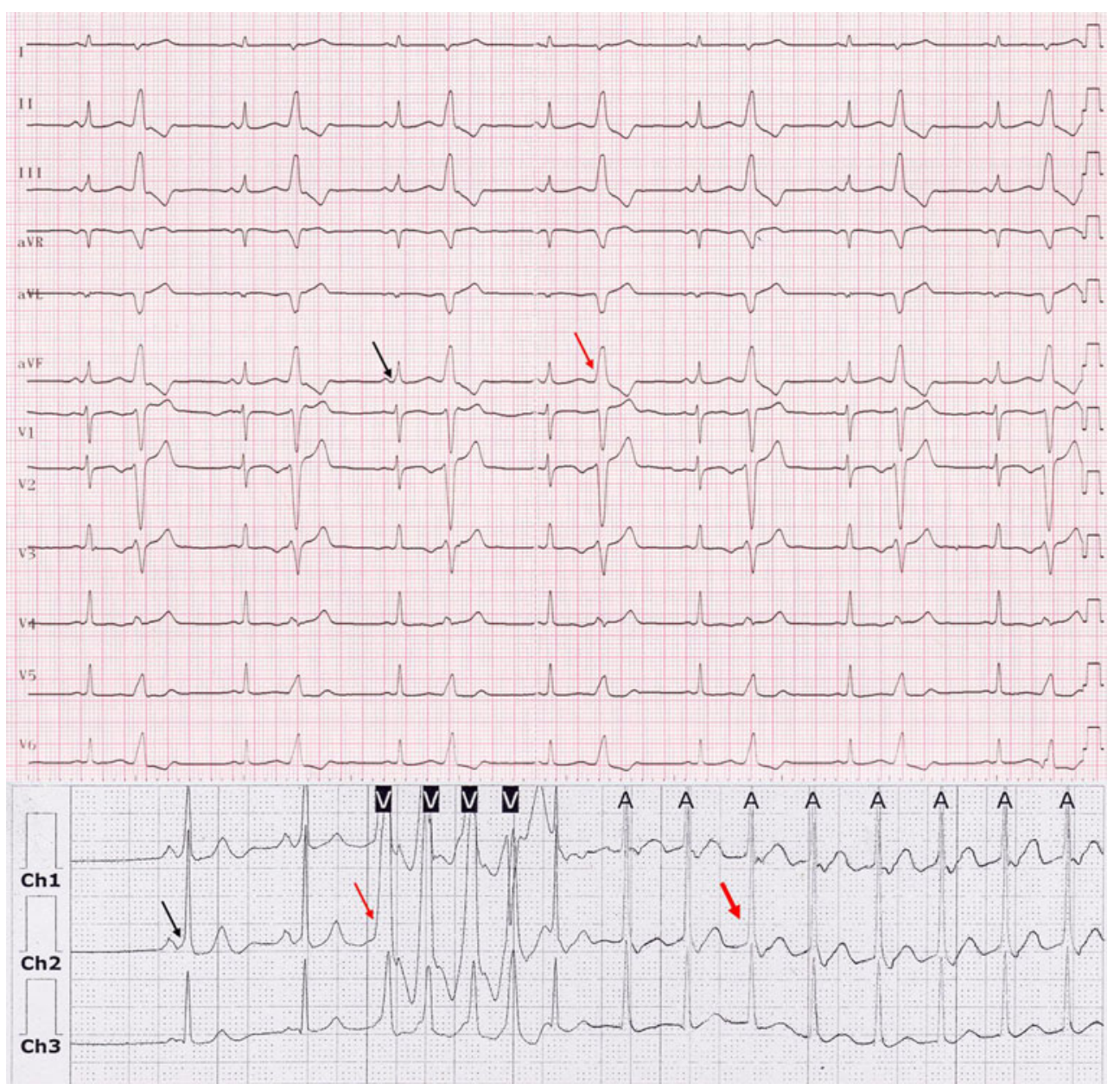




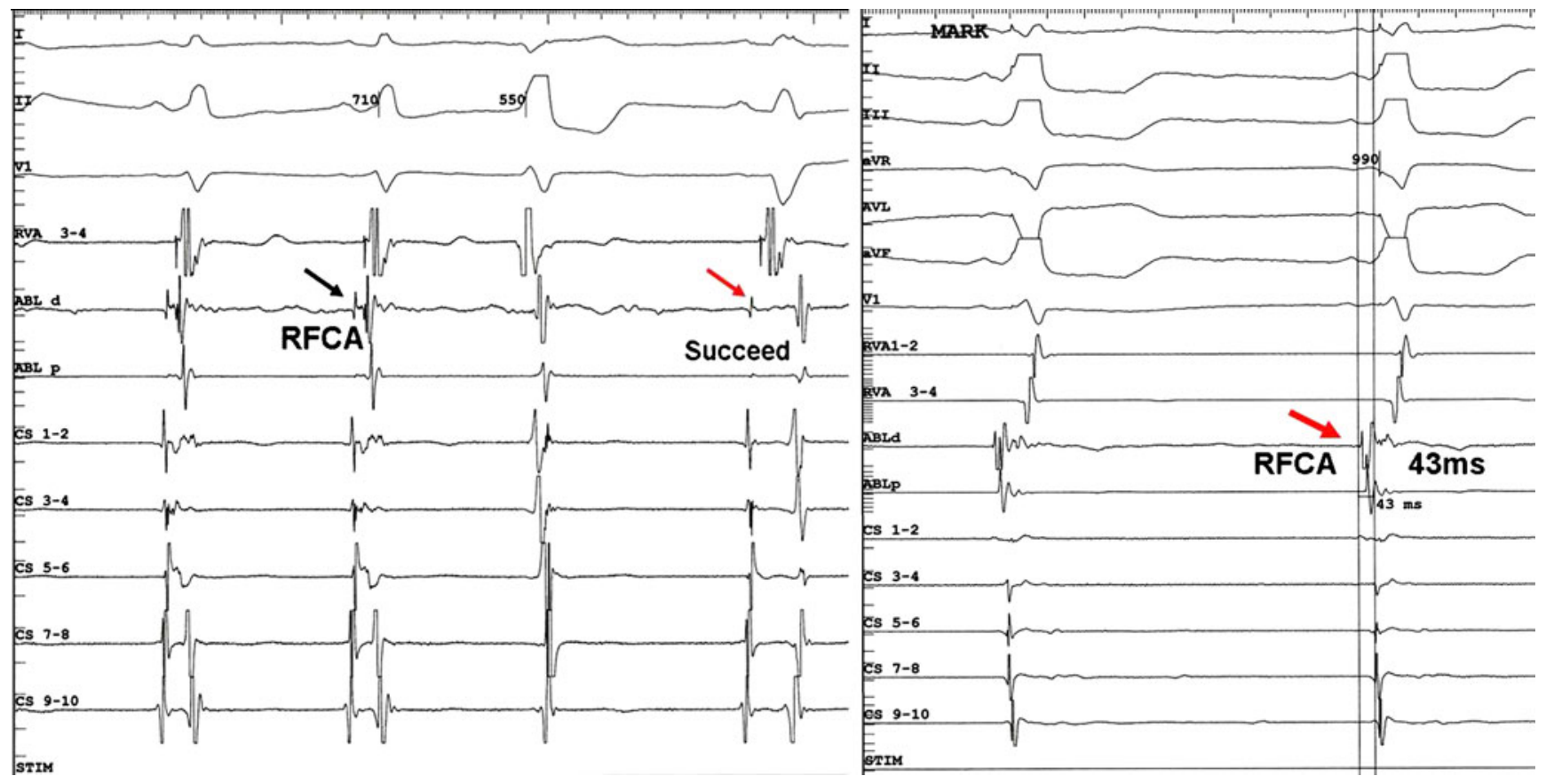

Fig. 2 Left panel the accessory pathway was successfully ablated. Black arrow $=$ delta wave, red arrow $=$ normal $\mathrm{P}$ wave. Paper speed is $50 \mathrm{~mm} / \mathrm{s}$. Right panel the original site of the PVCs at right ventricular outflow was successfully ablated. The PVC at the big-tip catheter excited $43 \mathrm{~ms}$ earlier than any other leads. Red arrow = target map. Paper speed is $50 \mathrm{~mm} / \mathrm{s}$
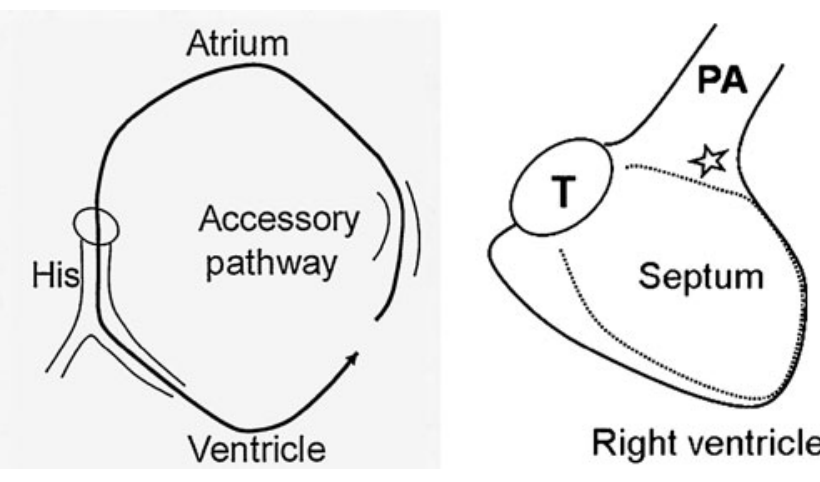

Right ventricle

Fig. 3 The drawing shows the ablated site of VT (right panel, asterisk ablated site) and the orthodromic circus movement tachycardia (left panel) 